\title{
Reply to the comments of $\mathbf{R}$. Klein on Advantages and challenges in coupling an ideal gas to atomistic models in adaptive resolution simulations
}

\author{
Karsten Kreis ${ }^{1,2, a}$, Aoife C. Fogarty ${ }^{1, b}$, Kurt Kremer $^{1, c}$, and Raffaello Potestio ${ }^{1, d}$ \\ 1 Max Planck Institute for Polymer Research, Ackermannweg 10, 55128 Mainz, Germany \\ 2 Graduate School Materials Science in Mainz, Staudinger Weg 9, 55128 Mainz, Germany
}

Abstract.

We thank R. Klein for his kind remarks and his interest in our work. He has raised some pertinent points in his comment, which we address in turn in this continuation of the discussion.

\section{The ideal gas model}

The work under consideration here concerns the coupling of an atomistic water model to an ideal gas via the Adaptive Resolution Simulation scheme (AdResS). ${ }^{1}$ Firstly, Klein considers our ideal gas model in the context of the kinetic theory of gases. In our work, by ideal gas we refer to non-interacting particles with vanishing cross-sectional area, and therefore no collisions between particles in the ideal gas region. In fact, in our implementation it is the thermostat which ensures the desired velocity distribution in the ideal gas bath.

\section{Why is the CG model needed at all?}

The only role of the coarse-grained (CG) region in the work we are currently discussing, and in the applications suggested therein, is to absorb and supply particles in a statistically correct fashion. (We point out that of course this is not necessarily the only role of the CG region in AdResS simulations in general.) As suggested by Klein, one could in theory simply create and destroy particles directly on the outer boundary of the hybrid (HY) region, according to an appropriate random distribution. However, the ideal gas bath used here has a negligible computational cost and provides a straightforward way to control variables such as the density, temperature and/or pressure of the bath, using existing methodological knowledge and computational implementations. Approaches with greater similarity to that suggested by Klein also exist, such as that of Grünwald and Dellago, ${ }^{2}$ in which ideal gas particles are inserted at the surface of a region of interest according to the Poisson distribution.

\section{Weighting scheme for interpolation}

Over the years, slight variations have developed in the weighting function used in the interpolation of potentials or forces in the AdResS methodology. This can be done using the product $w_{i} w_{j}$ (for convenience we call this Weighting Scheme 1 here) or the arithmetic average $\frac{w_{i}+w_{j}}{2}$ (Weighting Scheme 2), where $w_{i}$, also referred to as $\lambda_{i}$, is the switching function which controls the resolution of particle $i$ and which varies from 1 in the atomistic region to 0 in the coarse-grained region.

The AdResS scheme, first developed in its force-interpolation variant, has historically used weighting scheme $1 .^{3-5}$ It is also possible to formulate a potential interpolation using this weighting scheme, as done by Wang \& Agarwal in this issue. Weighting Scheme 2 was first used with the

\footnotetext{
a e-mail: kreis@mpip-mainz.mpg.de

b e-mail: fogarty@mpip-mainz.mpg.de

c e-mail: kremer@mpip-mainz.mpg.de

d e-mail: potestio@mpip-mainz.mpg.de
} 
potential interpolation. ${ }^{6}$ It is the scheme we use in our work in this issue, and follows naturally when the single-particle potentials are written as in Eq 3 in our paper. However, force interpolation could also be performed using this weighting scheme. ${ }^{7}$

In the case of the potential interpolation, the choice of Weighting Scheme 1 or 2 leads to a major difference in the expression for the intermolecular forces, namely in the drift force term, or the term which depends on the gradient of the weighting function and which breaks Newton's third law (final term in Eq 4 of Wang \& Agarwal, final term in Eq 4 of our paper). With Weighting Scheme 2, this is a one-particle term, while with Weighting Scheme 2, it is a two-particle term, thus complicating the theoretical interpretation of the drift force and the terms used to compensate it.

In the case of the force interpolation, the difference between the two weighting schemes becomes less critical and more a matter of taste. However, it should be pointed out that Weighting Scheme 2 has a symmetry which is lacking in Weighting Scheme 1. In Weighting Scheme 1, CG particles only ever have CG interactions, while with Weighting Scheme 2, CG particles close to the HY/CG boundary interact with HY particles via both the CG and the AT forces, in the same way that AT particles close to the AT/HY boundary interact with HY particles via both the CG and the AT forces. This can have implications for the computational implementation, or for the definition of buffer regions around the HY region where the interactions are not completely single-resolution. ${ }^{8}$

\section{Acknowledgments}

K. Kreis is recipient of a fellowship funded through the Excellence Initiative (DFG/GSC 266). K. Kremer and A. Fogarty acknowledge research funding through the European Research Council under the European Union's Seventh Framework Programme (FP7/2007-2013) / ERC grant agreement n. 340906-MOLPROCOMP. R. Potestio and K. Kremer acknowledge funding from the SFB-TRR 146 grant.

\section{References}

1. R. Potestio, C. Peter and K. Kremer, Entropy, 2014, 16, 4199-4245.

2. C. Dellago and M. Grünwald, Mol. Phys., 2006, 104, 3709-3715.

3. M. Praprotnik, L. Delle Site and K. Kremer, J. Chem. Phys., 2005, 123, 224106.

4. S. Matysiak, C. Clementi, M. Praprotnik, K. Kremer and L. Delle Site, J. Chem. Phys., 2008, 128, 024503 .

5. S. Fritsch, C. Junghans and K. Kremer, J. Chem. Theory Comput., 2012, 8, 398-403.

6. R. Potestio, S. Fritsch, P. Español, R. Delgado-Buscalioni, K. Kremer, R. Everaers and D. Donadio, Phys. Rev. Lett., 2013, 110, 108301.

7. K. Kreis, D. Donadio, K. Kremer and R. Potestio, EPL (Europhysics Letters), 2014, 108, 30007.

8. H. Wang, C. Schtte and L. Delle Site, J. Chem. Theory Comput., 2012, 8, 2878-2887. 